\title{
Systemic sclerosis and its oral health implications
}

\author{
Agata Puzio ${ }^{1, B, D-F}$, Brygida Przywara-Chowaniec ${ }^{2, B-F}$, Lidia Postek-Stefańska, ${ }^{1, D-F}$, \\ Katarzyna Mrówka-Kata ${ }^{3, B, D-F}$, Karolina Trzaska ${ }^{4, B, E}$ \\ ${ }^{1}$ Department of Pediatric Dentistry, School of Medicine with the Division of Dentistry in Zabrze, Medical University of Silesia, Katowice, Poland \\ $22^{\text {nd }}$ Department of Cardiology, School of Medicine with the Division of Dentistry in Zabrze, Medical University of Silesia, Katowice, Poland \\ ${ }^{3}$ Department of Otorhinolaryngology and Oncological Laryngology, School of Medicine with the Division of Dentistry in Zabrze, Medical University of Silesia, Katowice, Poland \\ ${ }^{4}$ Clinical Department of Internal Diseases, Dermatology and Allergology, School of Medicine with the Division of Dentistry in Zabrze, Medical University of Silesia, Katowice, \\ Poland
}

A - research concept and design; B - collection and/or assembly of data; $\mathrm{C}$ - data analysis and interpretation;

$D$ - writing the article; $E$ - critical revision of the article; $F$ - final approval of the article

\section{Address for correspondence \\ Agata Puzio}

E-mail:agata.puzio1@gmail.com

\section{Funding sources}

None declared

\section{Conflict of interest}

None declared

Received on January 1, 2017

Reviewed on March 22, 2017

Accepted on September 6, 2017

Published online on August 6, 2018

\begin{abstract}
Systemic sclerosis (SSc) is a chronic, generalized disorder of the connective tissue. It is characterized by immune disorders, abnormalities of morphology and functions of small blood vessels, and the presence of inflammatory process. The pathogenesis of this disorder has not yet been fully understood. The classification criteria were established by The American College of Rheumatology (ACR). A number of clinical types are distinguished due to the diversity of the clinical picture. These types are characterized by a different course, presence of organ complications and prognosis. Connective tissue disorders are interdisciplinary conditions and, therefore, the subject of interest of different medical specialties, including dentistry. The oral cavity may be the place of pathological manifestations within soft and hard tissues. Such manifestations are the results or the primary symptom of systemic diseases. The relationship between the health of the oral cavity and systemic diseases has been frequently reported in the literature. Lesions in the oral cavity in patients with SSc are discussed in detail in the present paper. Management includes the administration of drugs that prevent tissue ischemia and post-ischemic consequences as well as drugs that inhibit inflammatory-immune processes and excessive collagen production.
\end{abstract}

Key words: connective tissue diseases, oral manifestations, systemic scleroderma

Cite as

Puzio A, Przywara-Chowaniec B, Postek-Stefańska L,

Mrówka-Kata K, Trzaska K. Systemic sclerosis and its oral health implications. Adv Clin Exp Med. 2019;28(4):547-554. doi:10.17219/acem/76847

DOI

10.17219/acem/76847

\section{Copyright}

Copyright by Author(s)

This is an article distributed under the terms of the

Creative Commons Attribution Non-Commercial License

(http://creativecommons.org/licenses/by-nc-nd/4.0/) 


\section{Introduction}

Systemic sclerosis (SSc) is a chronic systemic connective tissue disease. It is characterized by immune disorders, disorders of morphology and functions of small blood vessels, and the presence of the inflammatory process. The predominant symptom of SSc is progressive fibrosis of the skin, organs and systems. The disease usually involves kidneys, lungs, gastrointestinal tract, as well as osteoarticular, cardiovascular and nervous system. Disease activity, the degree and the extent of internal organ involvement, and the prognosis differ among patients. ${ }^{1}$

The pathogenesis of SSc has not been fully understood. The factors considered include the genetic background, environmental factors, blood vessel malfunctions, loss of immune tolerance, impaired biosynthesis, degradation of the extracellular matrix of the connective tissue, and hormonal factors. ${ }^{1}$ It seems that in individuals with a certain genetic predisposition, some (toxic) environmental factors may result in blood vessel damage and immune cell activation, which leads to non-specific activation of fibroblasts and other connective tissue cells producing the extracellular matrix components. ${ }^{2}$

The incidence of SSc is higher in women as compared to men (F:M of 3:1). The risk of developing the disease in the general population ranges from $0.009 \%$ to $0.026 \%$. A positive family history is the most significant factor predisposing to the development of SSc, in which case the incidence risk is from 10 to 158 times higher compared to the general population. The peak incidence occurs between the $2^{\text {nd }}$ and the $5^{\text {th }}$ decade of life. ${ }^{3}$

The classification criteria of SSc were developed by the American College of Rheumatology (ACR). The presence of 1 large or 2 small criteria allows for the diagnosis of SSc. Large criteria are as follows: hardening of the skin covering the areas located proximally to the metacarpophalangeal joints and metatarsophalangeal joints. Small criteria are the following: sclerodactylia (hardening of the skin) distally to metacarpophalangeal joints, fingertip tissue loss and parabasal pulmonary fibrosis. ${ }^{4}$

In order to facilitate the early detection of the disease when clinical symptoms are not that developed or distinctive yet, a group of symptoms was distinguished that make up a high risk group for the development of SSc, known as early scleroderma. The most important symptom is Raynaud's phenomenon (paroxysmal pallor of fingers, feet, nose, or earlobes due to the contraction of small arteries and arterioles in response to cold, vibration or emotions) and the occurrence of microangiopathy (diagnosed based on nailfold capillaroscopy where the assessment includes the appearance and number of vessels, their morphology, and the presence of extravasation and edema) or the presence of specific autoantibodies in the serum - anti-centromere antibodies or anti-topoisomerase 1 antibodies. ${ }^{5}$

The diversity of the clinical picture of the disease allowed us to distinguish a number of clinical types that differ in terms of the disease course, the presence of organ complications and the prognosis. The distinguished forms are the following:

- systemic sclerosis with generalized hardening of the skin (diffuse systemic sclerosis (dSSc));

- scleroderma with limited hardening of the skin (limited systemic sclerosis (ISSc));

- transitional forms: dSSc/lSSc;

- systemic sclerosis without scleroderma;

- early SSc with limited scleroderma;

- scleroderma overlap syndromes;

- scleroderma in the course of cancer. ${ }^{6}$

\section{Generalized systemic sclerosis}

Generalized SSc is characterized by the rapid progression of symmetric hardening of the skin of proximal and distal parts of the extremities, face and trunk. Raynaud's phenomenon appears quickly (time between the occurrence of this symptom and a noticeable hardening of the skin is less than 1 year) and early involvement of internal organs is observed. This form of the disease has a very rapid course, especially in the initial period. The rapid progression of skin lesions and the involvement of internal organs (lungs, heart, kidneys) in the early stage of the disease indicate a particularly bad prognosis. In most cases, anti-topoisomerase $1 \mathrm{Scl}-70$ antibodies are detected. Capillaroscopy demonstrates characteristic vascularization.

\section{Limited systemic sclerosis}

Limited SSc is characterized by hardening of the skin in the areas distal to the elbows and knees. Typical changes involve facial skin. The majority of patients present with Raynaud's phenomenon, which precedes the onset of scleroderma. This form is characterized by a chronic, very slow, but steadily progressive course. Previously, it was described as the CREST syndrome, including soft tissue calcifications (calcinosis), Raynaud's phenomenon, esophageal dysfunction, sclerodactylia, and telangiectasia in the skin (hence the acronym). About $70-80 \%$ of patients with this form of the disease have anti-centromere antibodies, and a capillaroscopy shows vessel widening (mega capillaries).

\section{Systemic sclerosis without skin hardening}

Systemic sclerosis without skin hardening is also known as "scleroderma without scleroderma". In this disease entity, the involvement of organs and internal systems is observed, Raynaud's phenomenon is not always present and skin changes in the form of hardening do not occur. The results of serological tests are characteristic. 


\section{Scleroderma overlap syndromes}

The literature describes the cases of the coexistence of scleroderma and other connective tissue diseases, most often rheumatoid arthritis and systemic lupus erythematosus.

The major symptoms of SSc include the so-called tough skin and the presence of atrophic changes such as erosions, ulcers and foci of skin necrosis. Hardening of the skin is due to the accumulation of collagen fibers. The skin becomes smooth and thickened. The changes progress from the distal extremities. Limited systemic sclerosis refers to a disease where tightness primarily affects fingers, hands and distal forearms, feet and legs (hardening confined to skin usually from elbows distally and from knees distally); dSSc also includes hardening of the skin of proximal extremities and the trunk. In the initial stage of the disease, the skin is stretched, smooth and thinned ("too tight"). Muscle contractures with the claw-like hand stiffness are observed, and tendon fibrosis and restricted mobility occur as a consequence. ${ }^{7}$ As the disease progresses, clinical signs of internal organ involvement are reported. Gastrointestinal tract symptoms occurring in most patients with SSc are mainly related to motor impairment (impaired peristalsis of the oesophagus and colon, reduced sphincter tonus) and secretion impairment. ${ }^{5}$ Involvement of the respiratory system, affecting as many as $2 / 3$ of patients, is one of the most severe complications of scleroderma. In $16-22 \%$ of patients, it results in death. Interstitial lung disease (alveolitis and interstitial inflammation with secondary fibrosis) usually develops in the process of fibrosis. Primary and secondary pulmonary hypertension is usually detected in patients.

Cardiac involvement is noted in the majority of patients with generalized scleroderma and about $10 \%$ develop cardiomyopathy, resulting from cardiac fibrosis. Conduction disturbances are a common symptom of cardiac involvement. Typical symptoms also include pericarditis. Renal involvement is characterized by a scleroderma renal crisis, which is a newly developed renal failure or malignant hypertension in patients with SSc. Progressive renal failure sometimes forces dialysis.

Typical symptoms of the osteoarticular system are as follows: arthralgia of considerable intensity, edema and stiffness of fingers and knees. Symptoms may occasionally be similar to rheumatoid arthritis. Patients develop chronic arthritis with deposition of fibrin and joint contractures. Subcutaneous nodules may be formed around the joint area, especially in the hands, which results from the deposition of calcium compounds in the connective tissue. The reason of muscle weakness is due to muscle wasting, which is frequent in patients with extensive skin hardening. Muscular changes may be non-inflammatory, fibrosing myopathy with the dominance of myalgia, atrophy or typical cellulitis. ${ }^{2}$

Treatment of SSc is possible only in the active phase of the disease, which is a result of progressive inflammation.
Fibrotic-type changes are irreversible. The inhibition of pathologic changes is possible only at the early stage of the disease. Therapeutic treatment with the already present fibrosis is limited to the alleviation of symptoms and treatment of complications. Treatment of scleroderma is multi-directional and focused on the inhibition of the biosynthesis and the accumulation of fibrous proteins, the inhibition of dysfunction within the blood vessels and the reduction of inflammation. As the cause of the disease remains unknown, no causal treatment exists. Therefore, only symptomatic medications can be administered. There are no drugs effectively inhibiting or delaying the progression of the disease. The current trend in the treatment is known as organ-specific therapy, the aim of which is to protect an organ with possibly early treatment of pathologies and potential remodeling of the existing changes.

The following groups of drugs are suggested in the treatment of scleroderma ${ }^{8}$ :

- drugs preventing tissue ischemia and its effects (calcium channel blockers);

- drugs inhibiting inflammatory-immune processes (immunosuppressive drugs - cyclophosphamide, methotrexate, mycophenolate mofetil, cyclosporine A);

- drugs inhibiting excessive collagen production (D-penicillamine, colchicine).

The orofacial region remains the most affected by SSc in about $80 \%$ of cases. ${ }^{9}$ Oral abnormalities are frequently reported in SSc. They may, however, be overshadowed by other systemic symptoms of oral problems, and dental (decayed, missing teeth), periodontal and orofacial anomalies (e.g., xerostomia, mandibular bone resorption or microstomia). ${ }^{10}$

Skin involvement is noted in the majority of patients with SSc. Abnormal accumulation of connective tissue components in the skin results in the loss of dermal elasticity with subsequent dermal thickening and hardening.

The disease is characterized by the occurrence of 3 phases of skin thickening related to the facial area: edematous phase, indurative phase and atrophic phase. In the $1^{\text {st }}$ phase (mostly painless), (non)pitting edema may occur in the face and it may last terminally. The next phase is related to edema that is replaced by a markedly thickened skin and considerably thinned epidermal layer, which in consequence results in the loss of skin creases and a "choking out" of hair follicles, sweat and sebaceous glands. ${ }^{11}$ The skin is frequently pruritic with the observed loss of specialized skin structures, which contributes to changes in perspiration and also to the loss of hair growth. ${ }^{12}$

In the $3^{\text {rd }}$ phase the rapidly progressive skin fibrosis in consequence results in changes in the physical appearance. Deposition of subcutaneous collagen in facial skin leads to a smooth, tight, expressionless face, often termed as the "mask-like" appearance with the simultaneous disappearance of wrinkles and perioral furrows. ${ }^{9}$

Nasal alae may undergo atrophy, which may result in a "mouse-like" face. Other significant orofacial manifestations are connected with lacrimal gland fibrosis. 
Of ophthalmological manifestations, dry eyes with keratoconjunctivitis sicca or xerophthalmia are the most common. It is particularly problematic due to the fact that eyelid scarring leads to a chronic widening of palpebral fissures and also to inadequate eyelid closure, which contributes to further drying of the eyes. ${ }^{13}$

Other dermal manifestations include telangiectasia, frequently in the region around the mouth, cheeks, lips, and nose. Telangiectasia manifests as small red spots by edema of small blood vessels beneath the skin. Cosmetic problems are also noted due to facial occurrence of telangiectasia. ${ }^{14}$ Also, changes in the color of the skin are reported and hyper-and hypopigmentation may also be observed in the affected areas. ${ }^{12}$

Sclerosis of the lips and the skin around the mouth area causes reduced mouth opening (known as microstomia) and width (microcheilia) with the reduced interincisal distance. Microstomia may be related to problems with speaking or chewing. Additionally, oral hygiene and dental treatment is difficult due to the limited access to the teeth. The maximum mouth opening should be measured during the oral examination. The maximum interincisal opening of $40 \mathrm{~mm}$ or less should indicate the need for investigation regarding its etiology. ${ }^{14}$

Mouth-opening limitation and reduced cheek and tongue movement contribute to the deterioration in dental health. Oral and facial manifestations related to sclerodactylia may lead to a more rapid decline. Therefore, regular oral examination is of great importance. Maintenance of the existing dentition is significant due to the fact that microstomia can make the prosthetic replacement difficult. ${ }^{15}$

Mouth-stretching exercises are recommended as they reduce and decrease the risk of mouth-opening limitation. ${ }^{16}$ Some approaches and techniques connected with active exercises are recommended, and some authors reported success related to achieving expanded movements of the mandible. ${ }^{3}$

Perioral exercise programs need to be done on a regular basis. Combined treatments, including physical therapy, mouth stretching exercises or massage may contribute to the improvement in mouth opening.

Kinesiotherapy is based on specific passive (tongue depressors placed between the posterior teeth), active (thumb placement in opposite corners of the mouth with outward pulling) or assisted exercises to improve the opening of the mouth by means of stretching and exercises that increase mimic muscles mobility. ${ }^{17}$

Connective tissue massage is indicated to increase local bloodstream and to relax the involved tissue. Treatment of the neck and the clavear regions in the case of facial involvement of SSc should not be neglected. Kabat's method, which is a neurorehabilitation technique using movement patterns with proprioceptive facilitation techniques to strengthen neuromuscular recruitment, offers benefits.

Patients with SSc require a multidisciplinary and multitasking rehabilitation approach, which can be provided by an experienced team, including physicians and therapists. ${ }^{17}$ In severe cases, surgical procedures (e.g., bilateral commissurotomy or commissuroplasty) are indicated for the increase in mouth opening. ${ }^{18}$

Dermatological treatments (such as photodynamic therapy, phototherapy with UV long wavelength light or photochemotherapy) demonstrate benefits. ${ }^{16}$ Comstedt et al. in their pilot study, treated SSc patients with microstomia using intense pulse light (IPL), which is known for its ability to remodulate the collagen formation in the skin. Those scientists observed that IPL could increase dermal mobility around the mouth area and reported a significant post-treatment improvement in the interincisal distance. ${ }^{19}$

Bennani et al. reported that $\mathrm{CO}_{2}$ laser treatment of severe limited mouth opening in SSc patients was connected with significant improvement in the mouth opening 3 months after the $1^{\text {st }}$ laser application, with further improvement observed at 1 year. ${ }^{20}$

Onesti et al. in their study, used autologous fat transplantation and autologous adipose-derived stromal cells (ADSCs) injection into the perioral region. ${ }^{21}$ Their aim was to increase dermal mobility in that region. Autologous fat transplantations have recently become the first-choice technique to hide skin lesions. This approach, which uses the patient's own body fat as a natural filler to achieve structural modifications, decreases the rates of complications related to the foreign material.

Autologous adipose-derived stromal cells are characterized by the ability to differentiate into different types of cells under specific conditions. Advanced cellbased therapies offer promising therapeutic possibilities to improve both repair and regeneration of the damaged tissue.

Favorable outcome was reported in all patients treated with fat transplantation or ADSCs. Improvement was also reported in subjective wellness of the skin in the perioral areas. Post-treatment increased mouth opening was reported in patients. $^{21}$

The involvement of facial tissues and jaw muscles can also cause pressure and mandibular resorption, which is observed in approx. $10 \%$ of SSc patients and is related to pathological fractures. ${ }^{16}$ Resorption of the mandible is characterized by a multifactorial etiology. ${ }^{22}$ Erosions are the result of pressure exertion on the bone via the atrophic muscles at their attachment site. Other authors indicate that mandibular resorption can also be due to ischemia of the bone secondary to vasculitis. Ramon et al. indicated that the condyle, coronoid and mandibular angles areas that demonstrate resorption are supplied by small arterial branches of the internal maxillary artery rather than the main inferior alveolar artery. ${ }^{23}$ Systemic sclerosis may be more likely to affect these smaller arterial brunches, and in consequence leads to bone resorption secondary to diminished vascularity in those areas. Skin tightness that can result in pressure resorption of the bone, can be another factor in the resorption of the mandible. ${ }^{24}$ 
In maxillofacial regions, resorption is noted in attachment area of masticatory muscles (e.g., temporal, lateral pterygoid and anterior belly of digastric muscle). ${ }^{22}$ Mandibular angles are usually involved (37.6\%), followed by condyle $(20.8 \%)$, coronoid process $(20.0 \%)$ and the posterior border of the ramus (14.4\%). ${ }^{25}$ Mandibular bone resorption is commonly observed bilaterally. Blunting of mandibular angles can be visualized on orthopantomograph.

Mandibular erosions (beside condyle erosions) are mostly asymptomatic and are not related to any clinical symptoms. However, they could affect jaw appearance. ${ }^{26}$ They may develop in patients with the full or partial set of teeth. ${ }^{3}$ Extensive mandibular resorption increases the risk of mandibular fracture during tooth extraction. Therefore, each patient needs to be screened radiologically for early detection of mandibular resorption to prevent the occurrence of iatrogenic fracture. ${ }^{22}$ Marmary et al. suggested that angle resorption without other obvious cause could be considered pathognomic for SSc. ${ }^{27}$ Therefore, diagnostic diligence is required when evaluating patients with mandibular resorption, especially if asymptomatic. ${ }^{27}$

Scleroderma may occasionally be related to idiopathic tooth resorption. There are reports on external root resorption resulting from the erosive process influencing the adjacent mandible. ${ }^{29}$ Severe resorption can lead to painful trigeminal neuropathy (TN) caused by inferior alveolar nerve compression. ${ }^{9}$

The most frequent oral finding to precede systemic involvement appears to be TN characterized by short recurrent episodes of intense paroxysms of pain in the regions where 1 or more branches of the trigeminal nerve are distributed; TN may involve either 1 or both sides of the face. ${ }^{28}$ Sensory involvement is predominant in $\mathrm{TN}$ that rarely affects masticatory muscles. ${ }^{9}$ Of note, $\mathrm{TN}$ may be the very $1^{\text {st }}$ symptom of connective tissue diseases, and awareness of this possibility may allow for early and more accurate treatment. ${ }^{30}$ So far, 73 cases of TN have been documented, being the most frequently reported peripheral nervous system involvement in patients with SSc. ${ }^{31}$ Neuropathy is related to collagen deposition in the perineurium and/or reduced vascularity to the trigeminal nerve itself. ${ }^{32}$

Temporomandibular joint (TMJ) disorders are also frequently reported in SSc patients and could be the consequence of mandibular resorption. The prevalence of clinical signs of TMJ dysfunction (e.g., pain, TMJ sounds or impairment of mandibular movements) and typical magnetic resonance imaging (MRI) findings (disc, articular surface and bone changes) is higher in SSc patients in comparison to healthy controls. ${ }^{9}$

In SSc patients, the dysfunction of TMJ is likely due to the increased dermal thickness and decreased dermal elasticity caused by collagen deposition, which contributes to the restriction in jaw movements. ${ }^{16}$ Systemic scleroderma is characterized by symmetric, erosive synovitis, which can lead to joint irregularity and disability. Temporomandibular joint arthritis is a disease that is present in up to $80 \%$ of patients with rheumatic disease and causes pain, facial dysmorphism and lifetime disability if it is not properly early diagnosed. Matarese et al. assessed the detectable prevalence of TMJ symptoms and signs of dysfunction in SSc patients in comparison to healthy controls. ${ }^{33}$ One of the main results of that study was that SSc patients showed more symptoms and signs of TMJ dysfunction as compared to healthy controls. Magnetic resonance imaging in SSc patients revealed the following: disk without displacement, disk displacement with reduction, condyle flattening, erosions and irregularities, temporal eminence flattening, TMJ with degenerative bone changes, as well as osteophytes and condyle resorption. ${ }^{31}$

Temporomandibular joint changes in patients may not be caused only by SSc process. Differentiation between TMJ disorders affected by systemic disease and any other local pathology, including, e.g., osteoarthritis or traumatic arthritis, is often challenging. Magnetic resonance imaging should be applied to allow for a complete examination and aid differential diagnosis. ${ }^{34}$ High-resolution ultrasound (US) may be implemented as an alternative imaging technique to monitor disorders of TMJ in the case when MRI is not available. ${ }^{33}$

Another crucial oral finding is related to the involvement of oral mucosa. Submucosus fibrosis causes atrophy and then sensitivity of the oral mucosa, which becomes pale and sclerotic. Patients frequently report the burning mouth syndrome or dysesthesia. The sicca syndrome is noted in about $70 \%$ of patients, being secondary to salivary gland fibrosis. In 7-14\% of cases, patients may present Sjögren's syndrome.

Sclerosis and atrophy of the lips and perioral tissues can result in perioral streaks. With advanced sclerosis, lip retraction may become severe and patients may not be able to close the lips, hence mouth breathing, the sicca syndrome and chewing problems.

The tongue becomes smooth, shiny and hard. It is characterized by a considerably restricted mobility as a result of a reduced length of the frenulum and increased thickness. ${ }^{35}$ Initially, due to edema, it may increase in size. However, it later shrinks and is affected by fibrosis. The tongue frenulum is thickened, which may result in speech difficulty. The Raynaud's phenomenon of the tongue is very rarely observed in SSc patients. ${ }^{36}$

Oral mucosal telangiectasias, particularly in the area of the lateral border of the tongue and buccal mucosa of the cheek, are frequently reported. ${ }^{37}$ Gastrointestinal (GI) fibrosis is reported in about $90 \%$ of patients with scleroderma. Tightening of the perioral skin leads to decreased nutrient absorption. Destruction of the myenteric neurons causes oropharyngeal dysphagia and the risk of malnutrition. Additionally, associated xerostomia and microstomia may decrease oral intake. Esophageal dysfunction is considered to be the most common GI manifestation. Reduced lower esophageal sphincter tone is detected in up to $90 \%$ of the patients and, in consequence, may lead to significant 
reflux. Gastric dysmotility is also reported. Gastroparesis causes nausea and vomiting. The progressive nature of the disease and GI tract involvement may contribute to malnutrition. ${ }^{38}$ Malnutrition, gastroesophageal reflux disease (GERD), deficiencies in vitamins B9 and B12, or exocrine pancreatic insufficiency can exacerbate mucosal atrophy. ${ }^{9}$

Salivary gland hypofunction (SGH) and xerostomia are other frequently reported complaints. The prevalence of clinical xerostomia ranges from $22 \%$ to $70 \% .{ }^{10}$ Salivary gland fibrosis is detected around capillaries and excretory ducts. Capillary wall sclerosis causes functional abnormalities by reducing vascular permeability and periductal fibrosis impairs excretion of saliva. ${ }^{9}$ Salivary gland hypofunction is a condition with significantly reduced salivary flow, and can also lead to changes related to the chemical composition of saliva. Xerostomia is a subjective perception of dry mouth, which in fact may be accompanied by a reduction in salivary flow. The symptoms of the disease mostly include halitosis, oral soreness, altered taste, as well as difficulty in swallowing and talking. Both xerostomia and SGH increase the risk of caries, and contribute to periodontal diseases and oral infections, e.g., candidiasis. A decrease of the salivary $\mathrm{pH}$ due to xerostomia and GERD may be responsible for enamel and dentin erosion. ${ }^{9,39}$

Patients need to be checked for Sjögren's syndrome, since studies reported a $17-29 \%$ prevalence of this syndrome in scleroderma patients. ${ }^{14}$ Systemic sclerosis is defined as an autoimmune epithelitis characterised by lymphocytic infiltration of exocrine glands and epithelia in multiple sites, and it can be seen alone (primary SSc) or in association with other autoimmune rheumatic diseases (secondary SSc). ${ }^{40}$

Xerostomia may be caused by some drugs administered to SSc patients, e.g., cardiovascular, antidepressant or antihistamine drugs, diuretics, iron supplements, narcotic analgesics, daily aspirin, or anticholinergics. Assessment of dry mouth in all SSc patients should be made to prevent complications related to this condition.

Symptomatic relief of the dry mouth symptoms can include the following: regular water intake, sugar-free gums (topical salivary stimulants) or oral lubricants. In the case of patients with SGH artificial saliva substitutes, sialagogues (pilocarpine) or anetholtrithione may be of benefit. ${ }^{9,40,41}$

Pilocarpine-related adverse effects include, e.g., hyperhidrosis, nausea, rhinitis, dizziness, intestinal colic, and pollakiuria. The drug is contraindicated in cardiac patients or individuals with glaucoma, chronic obstructive pulmonary disease and asthma. ${ }^{42}$

In contrast to cholinergic agonist agents whose therapeutic effects last between 3 and 5 h, carboxymethyl cellulose salivary substitutes only provide palliative and shortlasting benefits. ${ }^{32}$

Dry mouth, decreased oral aperture and impairment in manual dexterity are responsible for dental plaque and increased risk of developing oral diseases, e.g., plaque-induced gingivitis. ${ }^{43}$ Several case-control studies demonstrated that patients with SSc present with more severe gingivitis compared to controls. Baron et al. indicated that SSc patients have significantly worse dental health (more missing teeth, more periodontal disease) in comparison to controls. ${ }^{10,44}$ Wood et al. found that more patients with SSc presented with periodontal pockets compared to healthy controls. ${ }^{45}$ The cause of gingivitis may be connected to the defective vascularity and changes in the microcirculation of the gingival tissues, which results in gingival bleeding. Felder et al. and Poole et al. suggested that impaired manual dexterity was connected with poor oral hygiene. ${ }^{46-48}$ Baron et al. indicated that decreased saliva, GERD and diminished hand function (influencing the ability to maintain good oral hygiene) are related to missing teeth. ${ }^{43}$ Reports confirm that SSc patients have higher periodontal indices (plaque index, gingival index) compared to controls. ${ }^{9}$ Leung et al. noticed that SSc patients experience a higher percentage bleeding on probing (BOP) and have deeper probing depth (PD) as compared to healthy subjects. ${ }^{47}$ Recently, a positive correlation between SSc and periodontitis has been reported.

Some authors reported a widening of periodontal ligament space (PDL) in patients with SSc, which is one of the most frequent radiological findings and may be considered one of the first radiographic signs of the disease. ${ }^{24}$ Further studies are needed to evaluate whether the radiographic image can be used as a potential diagnostic marker. Periodontal ligament space thickening can be noted in each group of teeth, with the most frequent involvement of the posterior areas. Baron et al. reported that PDL widening was connected with the severity of the disease. ${ }^{26}$ Periodontal ligament space is a soft connective tissue located between tooth roots and the inner wall of the alveolar socket, and connects the cementum to both gingival and alveolar bone. Its normal width ranges from 0.15 to $0.21 \mathrm{~mm}$. The reasons for PDL widening in SSc patients are still unknown, but considering the high fibroblast and collagen content of the PDL, it is not surprising that, as elsewhere in the body, the PDL fibroblasts may be activated, thus producing excess collagen which, consequently, may lead to ligament widening. The clinical consequences of a wide PDL remain unknown. No association has been reported between a wide PDL and missing teeth. ${ }^{26}$

Prevention of caries and periodontal disease is the prime objective in the treatment plan for SSc patients. Patient education is significant, with particular attention paid to mouth and dental hygiene, including brushing techniques and mouthwash with antiseptic agents. Powered toothbrushes may contribute to the improvement in oral hygiene. Most patients will need a compact head or pediatric toothbrush to gain maximum access. In patients with hand deformities and functional limitations, the extension of the toothbrush handle may facilitate the removal of plaque biofilm. ${ }^{32}$ Patients also need to be educated and instructed on the significance of flossing 
at least twice a day, with consideration given to adapted flossing (i.e., flossing devices that are hand-held). ${ }^{43}$ For those patients who do not floss due to impaired manual dexterity, oral irrigators or non-alcohol mouth rinse may be of benefit. ${ }^{48}$

Regular follow-up visits (every $2-3$ months) are recommended to control oral infection. ${ }^{32}$ Patients should have radiographic examination before immunosuppressive treatment in order to remove potential sources of odontogenic infections. ${ }^{9}$

Prevention of oral candidiasis is connected with cleaning removable prostheses. Special caution should be taken in the case of patients on anticoagulant therapy if antifungal treatment is to be implemented. Measurement of International Normalized Ratio (INR) is obligatory.

Prevention of caries is connected with fluoridation of the enamel with toothpaste and the application of fluoride preparations every 3 months. Panoramic radiography should be used for the early detection of caries to avoid invasive treatment. The dentist can perform conventional dental treatment in SSc patients quadrant by quadrant so as to avoid long visits.

In patients who undergo extraction, antibiotic should be administered 1 day before and their administering should be continued until complete scarring.

Before the initiation of the treatment with bisphosphonates, any unsalvageable teeth should undergo extraction and all invasive procedures should be completed due to an increased risk of osteonecrosis. Oral care can be given under local anesthesia (3\% lidocaine). It should be remembered that adrenaline use is to be avoided as it can worsen the microangiopathy.

A number of drug-related adverse events are reported, which are connected to the mouth and gum mucosa. These are the following:

- calcium-channel blockers may cause gingival hyperplasia;

- corticosteroids may induce mouth infections, e.g., oral candidiasis;

- cyclophosphamide also causes mouth infections or stomatitis when administered in high doses;

- methotrexate may be responsible for mouth ulcers;

- antibiotic administration may cause oral candidiasis;

- anticholinergic antidepressants may contribute to the exacerbation of the dry mouth syndrome;

- bisphosphonates may lead to jaw osteonecrosis;

- anti-vitamin K anticoagulants may provoke (spontaneous or induced) gingival bleeding. ${ }^{49}$

Prosthetic treatment by conventional approaches might be challenging due to microstomia. Due to the fact that patients report problems with insertion and removal of the prosthesis, it is necessary to fabricate complete and partial dentures different from the standard ones. ${ }^{50}$ Fractionated imprints must be applied. Sectional, collapsible or sectional-collapsible are the types of prostheses recommended for prosthodontics management in patients in whom limited intraoral access is observed. Two segments of sectional dentures can be fixed by means of various systems (clasp retainers, stud or magnetic or swing-lock attachments or pins, and a telescope system).

The use of soft liner is reported for the management of edentulous patients who complain of chronic pain, soreness or discomfort related to prolonged contact between the rigid denture base and the underlying tissues. ${ }^{50,51}$

In conclusion, patients with SSc are exposed to a number of health problems, among which dental issues deserve special attention. These patients should obtain dental care immediately after the diagnosis is established. Early implementation of preventive and curative actions enables the maintenance of oral health. The knowledge of clinical symptoms and of the dynamics of the disease process will make it easier for dentists to apply appropriate treatment. Attention should be firstly paid to the prevention of oral diseases (caries prevention and prevention of the diseases related to the mucous membrane, gingiva and periodontium). Conservative and surgical treatment, and also prosthetic rehabilitation should be performed by a specialized team of doctors based on interdisciplinary cooperation and the latest scientific knowledge.

\section{References}

1. Kuźnik-Trocha K, Winsz-Szczotka K, Komosińska-Vassev K, Olczyk K. Twardzina układowa: Etiopatogeneza, diagnostyka, leczenie. Diagn Lab. 2012;48(4):441-451.

2. Szymańska E, Maj M, Rudnicka L. Twardzina układowa: Przebieg kliniczny i możliwości terapeutyczne. Przegl Lek. 2005;62(12):1538-1541.

3. Marcucci M, Abdala N. Clinical and radiographic study of orofacial alterations in patients with systemic sclerosis. Braz Oral Res. 2009; 23(1):82-88.

4. Meissner M. Choroby tkanki łącznej: Co lekarz domowy powinien wiedzieć? Nowa Klin. 2009;16(1-2):21-31.

5. Więsik-Szewczyk W, Olesińska M. Współczesny obraz twardziny układowej. Pol Merkur Lekarski. 2010;28(167):416-420.

6. Krasowska D. Patogeneza, kryteria rozpoznawania i klasyfikacja twardziny układowej. Dermatologica. 2008;1(49):21-29.

7. Burkhart NW. Scleroderma. Registered Dental Hygienist. 2009;29(6): 58-61.

8. Sierakowski S, Kowal-Bielecka O, Gindzieńska-Sieśkiewicz E. Twardzina układowa. Reumatologia. 2004;42(Suppl 1):59-69.

9. Jung S, Martin T, Schmittbuhl M, Huck O. The spectrum of orofacial manifestations in systemic sclerosis: A challenging management. Oral Dis. 2017;23(4):424-439.

10. Baron M, Hudson M, Tatibouet, et al. The Canadian systemic sclerosis oral health study: Orofacial manifestations and oral health-related quality of life in systemic sclerosis compared with general population. Rheumatology (Oxford). 2014;53(8):1386-1394.

11. Perno GM. Invisible illnesses: When the body turns on itself. CJDH. 2008;23(3):9-14.

12. Dghoughi S, El Wady W, Taleb B. Systemic sclerosis case report and review of literature. N Y State Dent J. 2010;76(3):30-35.

13. Albilia JB, Lam DK, Blanas N, Clokie CM, Sandor GK. Small mouths, big problem? A review of scleroderma and its oral health implications. J Can Dent Assoc. 2007;73(9):831-836.

14. Chu CH, Yeung CM, Lai IA, Leung WK, Mok MY. Oral health of Chinese people with systemic sclerosis. Clin Oral Investig. 2011;15(6):931-939.

15. Cazal C, Sobral AP, Neves RF, Freire Filho FW, Cardoso AB, da Silveira MM. Oral complaints in progressive systemic sclerosis: Two cases reports. Med Oral Patol Oral Cir Bucal. 2008;13(2):114-118.

16. Ferreira E, Christmann RB, Borba EF, Borges CT, Siqeira JT, Bonfa E. Mandibular function is severely impaired in systemic sclerosis patients. J Orofac Pain. 2010;24(2):197-202. 
17. Maddali Bongi S, Del Rosso A, Gallucio F, et al. Efficacy of a tailored rehabilitation program for systemic sclerosis. Clin Exp Rheumatol. 2009;27(3 Suppl 54):44-50.

18. Dixit S, Kalkur C, Sattur AP, Bornstein MM, Felton F. Scleroderma and dentistry: Two case reports. J Med Case Rep. 2016;10(1):297.

19. Comstedt L, Svensson A, Hesselstrand R, Lehti L, Troilius Rubin A. Effects of intense pulsed light in microstomia in patients with systemic sclerosis: A pilot study. J Cosmet Laser Ther. 2017;19(3):143-148.

20. Bennani I, Lopez R, Bonnet D, et al. Improvement of microstomia in scleroderma after carbon dioxide laser treatment. Case Rep Dermatol. 2016;8(2):142-150.

21. Onesti MG, Fioramonti P, Carella S, Fino P, Marchese C, Scuderi N. Improvement of mouth functional disability in systemic sclerosis patients over one year in a trial of fat transplantation versus adiposederived stromal cells. Stem Cells Int. 2016;2016:2416192. doi:10.1155/ 2016/2416192

22. Sarala K, Jayachandran S, Kumar SS. Mandibular resorption in systemic sclerosis. Indian J Rheumatol. 2016;11(1):55-56.

23. Ramon Y, Samra H, Oberman M. Mandibular condylosis and apertognathia as presenting symptoms in progressive systemic sclerosis (scleroderma). Pattern of mandibular bony lesions and atrophy of masticatory muscles in PSS, presumably caused by affected muscular arteries. Oral Surg Oral Med Oral Pathol. 1987;63(3):269-274.

24. Anbiaee N, Tafakhori Z. Early diagnosis of progressive systemic sclerosis (scleroderma) from a panoramic point of view: Report of three cases. Dentomaxillofac Radiol. 2011;40(7):457-462.

25. Auluck A, Pai KM, Shetty C, Shenoi SD. Mandibular resorption in progressive systemic sclerosis: A report of three cases. Dentomaxillofac Radiol. 2005;34(6):384-386.

26. Baron $M$, Hudson $M$, Dagenais $M$, et al. Relationship between disease characteristics and oral radiologic findings in systemic sclerosis: Results from a Canadian Oral Health Study. Arthritis Care Res (Hoboken). 2016;68(5):673-680.

27. Marmary Y, Glaiss R, Pisanty S. Scleroderma: oral manifestations. Oral Surg Oral Med Oral Pathol. 1981;52(1):32-37.

28. Maclntosh RB, Shivapuja PK, Naqvi R. Scleroderma and the temporomandibular joint: Reconstruction in 2 variants. J Oral Maxillofac Surg. 2015;73(6):1199-1210.

29. de Figueiredo MA, de Figueiredo JA, Porter S. Root resorption associated with mandibular bone erosion in a patient with scleroderma. J Endod. 2008;34(1):102-103.

30. Nascimento IS, Bonfa E, de Carvalho JF, et al. Clues for previously undiagnosed connective tissue disease in patients with trigeminal neuralgia. J Clin Rheumatol. 2010;15(5):205-208.

31. Amaral TN, Peres FA, Lapa AT, Marques-Neto JF, Appenzeller S. Neurologic involvement in scleroderma: A systemic review. Semin Arthritis Rheum. 2013:43(3):335-347.

32. Tolle SL. Scleroderma: Considerations for dental hygienists. Int J Dent Hyg. 2008;6(2):77-83.

33. Matarese G, Isola G, Alibrandi A, et al. Occlusal and MRI characterizations in systemic sclerosis patients: A prospective study from Southern Italian cohort. Joint Bone Spine. 2016;83(1):57-62.
34. Aliko A, Ciancaglini R, Alushi A, Tafaj A, Ruci D. Temporomandibular joint involvement in rheumatoid arthritis, systemic lupus erythematosus and systemic sclerosis. Int J Oral Maxillofac Surg. 2011;40(7): 704-709.

35. Świerkot J, Kukiz-Świerkot G. Zmiany obserwowane na śluzówkach jamy ustnej w przebiegu układowych chorób tkanki łącznej. Przew Lek. 2000;3(7):73-77.

36. Gremain V, Richard L, Langlois V, Marie I. Raynaud's phenomenon of the tongue. Joint Bone Spine. 2017;84(2):231.

37. Philipone $\mathrm{E}$, Yoon AJ, Zegarelli D. Intraoral telangiectasias associated with Raynaud disease: A report of two cases. Quintessence Int. 2010;41(1):17-20.

38. Bharadwaj S, Tandon P, Gohel T, et al. Gastrointestinal manifestations, malnutrition, and role of enteral and parenteral nutrition in patients with scleroderma. J Clin Gastroenterol. 2015;49(7):559-564.

39. Yuen HK, Weng Y, Reed SG, Summerlin LM, Silver RM. Factors associated with gingival inflammation among adults with systemic sclerosis. Int J Dent Hyg. 2014;12(1):55-61.

40. Vitali C, Bombardieri S, Jonsson R, et al; European Study Group on Classification Criteria for Sjögren's Syndrome. Classification criteria for Sjögren's syndrome: A revised version of the European criteria proposed by the American-European Consensus Group. Ann Rheum Dis. 2002;61(6):554-558.

41. Hopcraft MS, Tan C. Xerostomia: An update for clinicians. Aust Dent J. 2010;55(3):238-244.

42. Saleh J, Figueiredo MA, Cherubini K, Salum FG. Salivary hypofunction: An update on aetiology, diagnosis and therapeutics. Arch Oral Biol. 2015;60(2):242-255.

43. Yuen HK, Hant FN, Hatfield C, Summerlin LM, Smith EA, Silver RM. Factors associated with oral hygiene practices among adults with systemic sclerosis. Int J Dent Hyg. 2014;12(3):180-186.

44. Baron M, Hudson M, Tatibouet S, et al. Relationship between disease characteristic and orofacial manifestations in systemic sclerosis: Canadian Systemic Sclerosis Oral Health Study III. Arthritis Care Res (Hoboken). 2015;67(5):681-690.

45. Wood RE, Lee P. Analysis of the oral manifestations of systemic sclerosis (scleroderma). Oral Surg Oral Med Oral Pathol. 1988;65(2):172-178.

46. Felder R, Reveal M, Lemon S, Brown C. Testing toothbrushing ability of elderly patients. Spec Care Dentist. 1994;14(4):153-157.

47. Leung WK, Chu CH, Mok MY, Yeung KW, Ng SK. Periodontal status of adults with systemic sclerosis: Case control study. J Periodontol. 2011;82(8):1140-1145.

48. Poole JL, Brewer C, Rossie K, Good CC, Conte C, Steen V. Factors related to oral hygiene in persons with scleroderma. Int J Dent Hyg. 2005; 3(1):13-17.

49. Alantar A, Cabane J, Hachulla E, et al. Recommendations for the care of oral involvement in patients with systemic sclerosis. Arthritis Care Res (Hoboken). 2011;63(8):1126-1133.

50. Singh K, Gupta N, Gupta R, Abrahm D. Prosthetic rehabilitation with collapsible hybrid acrylic resin and permanent silicone soft liner complete denture of a patient with scleroderma-induced microstomia. J Prosthodont. 2014;23(5):412-416.

51. Yenisey M, Kulunk T, Kurt S, Ural C. A prosthodontics management alternative for scleroderma patients. J Oral Rehabil. 2005;32(9):697-700. 